\title{
Emotion Recognition in EEG Signals Using Decision Fusion Based Electrode Selection
}

\author{
Himanshu KUMAR ${ }^{\mathrm{a}, 1}$, Nagarajan GANAPATHY ${ }^{\mathrm{b}}$, Subha D PUTHANKATTIL ${ }^{\mathrm{c}}$ and \\ Ramakrishnan SWAMINATHAN ${ }^{\mathrm{a}}$ \\ ${ }^{a}$ Biomedical Engineering Group, Department of Applied Mechanics, Indian Institute of \\ Technology Madras, Chennai, India \\ ${ }^{\mathrm{b}}$ Peter L. Reichertz Institute for Medical Informatics of TU Braunschweig and \\ Hannover Medical School, Germany \\ ${ }^{\mathrm{c}}$ Department of Electrical Engineering, National Institute of Technology Calicut, \\ Kozhikode, India
}

\begin{abstract}
Emotions are essential for the intellectual ability of human beings defined by perception, concentration, and actions. Electroencephalogram (EEG) responses have been studied in different lobes of the brain for emotion recognition. An attempt has been made in this work to identify emotional states using time-domain features, and probabilistic random forest based decision fusion. The EEG signals are collected for this from an online public database. The prefrontal and frontal electrodes, namely Fp1, Fp2, F3, F4, and Fz are considered. Eleven features are extracted from each electrode, and subjected to a probabilistic random forest. The probabilities are employed to Dempster-Shafer's (D-S) based evidence theory for electrode selection using decision fusion. Results demonstrate that the method suggested is capable of classifying emotional states. The decision fusion based electrode selection appears to be most accurate (arousal F-measure $=77.9 \%$ ) in classifying the emotional states. The combination of Fp2, F3, and F4 electrodes yields higher accuracy for characterizing arousal $(65.1 \%)$ and valence $(57.9 \%)$ dimension. Thus, the proposed method can be used to select the critical electrodes for the classification of emotions.
\end{abstract}

Keywords. Electroencephalography, Emotions, Probabilistic Random Forest, Decision Fusion

\section{Introduction}

Emotion is a dynamic pattern of response, involving elements of experience, actions, and physiology, through which an organism tries to cope with a matter or occurrence that is personally relevant. Recognition of emotions is the mechanism by which human emotions are identified [1].

In two main classes, discrete and dimensional, emotion is categorically studied. In the discrete model, the emotions are sub-divided into six categories: happy, sadness, fear, joy, anger and disgust. In the dimensional model, the mood states are placed in multidimensional space. Most common is the circumplex model of affect which utilizes a two-

${ }^{1}$ Corresponding Author; Himanshu Kumar, Biomedical Engineering Group, Department of Applied Mechanics, Indian Institute of Technology Madras, Chennai 600036, India, E-mail: him241994@gmail.com. 
dimensional plane - Valence (High and Low - HV, LV) and Arousal (High and Low HA, LA) [2].

Several studies have been reported for emotion recognition using Electroencephalogram (EEG) signals. Previous studies conducted using neurophysiological and functional neuroimaging methods have reported the association of the frontal lobe for emotion recognition [3]. It has also been established that the prefrontal cortex has a role in emotion modulation [4]. Time-domain features proved to be straightforward and useful in classification tasks. Earlier literature have reported the use of time domain-based features like mean, median, zero crossing, minima, and maxima for recognizing emotions using EEG signals [5]. Probabilistic approach, a mathematical method has been designed to increase the performance of classification. The Dempster-Shafer (D-S) theory is a tool for data fusion which can be used to combine various information from sources like sensors, which helps in handling imprecision, uncertainty, and incompletion [6].

In this work an attempt has been made to classify arousal and valence emotional states from EEG signal of five frontal electrodes, namely Fp1, Fp2, F3, F4, and Fz. Timedomain features and information fusion based upon the D-S theory at the decision level is employed to achieve reliable and accurate evaluation of critical channels. Classification of the emotions are carried out using probabilistic random forest classifier.

\section{Methods}

\subsection{Database Description}

The EEG signals from a publicly available DEAP database are used in this study [9]. It consists of 1280 multiple physiological signals, such as EEG, EMG, PPG, and EDA, from 32 normal subjects while watching 40 different audio-video recordings of 60 s. For this study, all the available data of the preprocessed EEG signals, (sampling rate of 128 $\mathrm{Hz}$ ) are considered. The signals had been annotated independently using the participant's experience with the stimulus in the arousal-valence dimension [7].

\subsection{Feature Extraction}

Eleven time-domain features are extracted from the preprocessed EEG signals obtained from prefrontal and frontal regions. The selected electrodes are namely - Fp1, Fp2, F3, $\mathrm{F} 4$, and Fz. The mathematical expression for time-domain features is given below.

RMS: It is estimated as the square root of the mean of a squared signal [8]. It is estimated as Eq. (1).

$$
R M S=\sqrt{\frac{1}{N} \sum_{t=1}^{N} x^{2}(t)}
$$

Where $\mathrm{N}$ is the total number of samples and $x(t)$ represents the signal.

Hjorth developed features, namely Activity, Mobility, and Complexity, to analyze signals [9].

Activity: It represents the power of the signal. 


$$
A_{\xi}=\frac{\sum_{t=1}^{T}(\xi(t)-\mu)^{2}}{T}
$$

Mobility: It represents ratio of standard deviation of the power spectrum.

$$
M_{\xi}=\sqrt{\frac{\operatorname{var}(\dot{\xi}(t))}{\operatorname{var}(\xi(t))}}
$$

Complexity: It estimates the bandwidth of a signal.

$$
C_{\xi}=\frac{M(\dot{\xi}(t))}{M(\xi(t))}
$$

Zero Crossings: The number of baseline crossing is computed in a fixed interval [10]. Minima and Maxima: It represents the maximum and minimum value of an EEG signal in a given time interval [10].

Mean of absolute values of first difference [11]:

$$
\delta_{X}=\frac{1}{N-1} \sum_{N-1}^{n=1}|X(n+1)-X(n)|
$$

Mean of absolute values of the first difference of normalized EEG [11]:

$$
\bar{\delta}_{X}=\frac{1}{N-1} \sum_{N-1}^{n=1}|\bar{X}(n+1)-\bar{X}(n)|
$$

$\bar{X}(n)$ represents the signal normalized to zero mean and unit variance.

Mean of absolute values of the second difference [11]:

$$
\gamma_{X}=\frac{1}{N-2} \sum_{N-2}^{n=1}|X(n+2)-X(n)|
$$

Mean of absolute values of the second difference of normalized EEG [11]:

$$
\bar{\gamma}_{X}=\frac{1}{N-2} \sum_{N-2}^{n=1}|\bar{X}(n+2)-\bar{X}(n)|
$$

\subsection{Decision fusion and Electrode Selection}

Probabilistic Random Forest (PRF) is used for the estimation of the probabilistic output of the classification. It is a modified version of the Random Forest (RF) algorithm [12]. To combine multiple classification outcomes obtained from different PRF models, a data fusion method is used. The D-S evidence theory as one of the data fusion method, with the benefits of addressing uncertainties by quantifying the degree of belief is used [13].

Decision fusion-based probability is obtained by classification probability from individual electrodes with PRF using leave one out cross-validation. Combined probability is then cross-referenced with the probabilities from the individual electrodes for the selection of the electrodes for classification. The classification performance of three electrodes with similar probability to fusion probability is compared with the performance of all five electrodes [13]. 


\section{Results}

Figure 2 shows the representative EEG signal from the Fp1 electrode for the HA, LA, $\mathrm{HV}, \mathrm{LV}$. It is seen that the amplitude inLA is higher as compared to the HA. Similarly, the amplitude in LV is higher as compared to the HV.

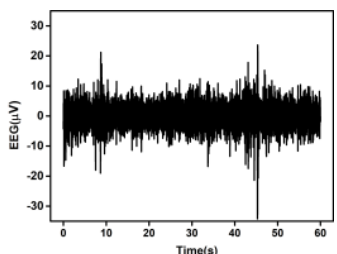

(a)

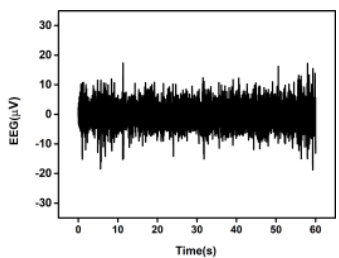

(c)

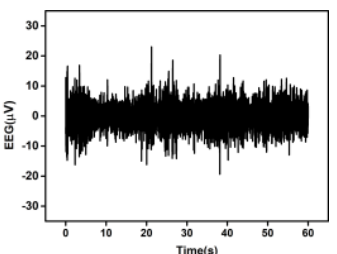

(b)

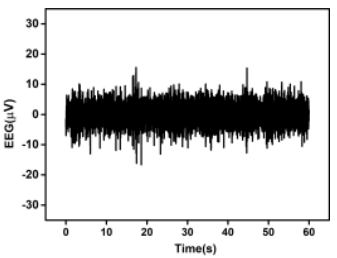

(d)

Figure 1. Representative EEG signal for low LA (a), HA (b), LV (c), and HV (d) of a subject for a representative electrode Fp1

Table 1. Classification performance by random forest classifier from individual electrode

\begin{tabular}{llllll}
\hline Scale & Electrode & Precision & Recall & F-1 Score & Accuracy \\
\hline \multirow{5}{*}{ Arousal } & Fp1 & 0.643 & 0.973 & 0.774 & 0.641 \\
& Fp2 & $\mathbf{0 . 6 4 5}$ & 0.946 & 0.767 & 0.637 \\
& F3 & 0.643 & 0.979 & $\mathbf{0 . 7 7 6}$ & $\mathbf{0 . 6 4 4}$ \\
& F4 & 0.632 & $\mathbf{0 . 9 8 9}$ & 0.771 & 0.630 \\
Valence & Fz & 0.638 & 0.963 & 0.768 & 0.632 \\
& Fp1 & 0.428 & 0.240 & 0.308 & 0.501 \\
& Fp2 & 0.479 & 0.208 & 0.290 & 0.530 \\
& F3 & 0.495 & 0.262 & 0.343 & 0.536 \\
& F4 & $\mathbf{0 . 5 5 2}$ & $\mathbf{0 . 4 2 1}$ & $\mathbf{0 . 4 7 8}$ & $\mathbf{0 . 5 7 5}$ \\
\hline
\end{tabular}

Table 1 shows the classification performance of individual electrodes using PRF. The top three F-1 scores for arousal are obtained from F3, Fp1 and F4 electrodes with F1score $0.776,0.774$, and 0.771 respectively. Similarly for valence, the top three F-1 scores are F4, Fz and F3 electrodes with F-score as 0.478, 0.404, and 0.343.

The classification performance of all the combined five channels and fusion based selected channels are represented in Table 2. It is seen that the selected channels yields higher performance $(\mathrm{F}-1 \mathrm{Score}=0.779$ for arousal and 0.470 for valence dimension).

Table 2. Classification performance for arousal and valence dimension for all and selected electrodes

\begin{tabular}{llllll}
\hline Electrodes & Dimesion & Precision & Recall & F-1 Score & Accuracy \\
\hline All & Arousal & 0.648 & $\mathbf{0 . 9 7 8}$ & 0.779 & 0.650 \\
& Valence & 0.538 & 0.376 & 0.442 & 0.562 \\
\hline \multirow{2}{*}{ Selected } & Arousal & $\mathbf{0 . 6 4 9}$ & 0.975 & 0.779 & $\mathbf{0 . 6 5 1}$ \\
& Valence & $\mathbf{0 . 5 6 1}$ & $\mathbf{0 . 4 0 4}$ & $\mathbf{0 . 4 7 0}$ & $\mathbf{0 . 5 7 9}$ \\
\hline
\end{tabular}




\section{Discussion}

The variation is amplitude in different EEG emotion signal might be due to the intensity of the emotions. The F3 yields the highest accuracy in arousal, while F4 yields the highest accuracy in the valence dimension (see Table 2). The highest precision is obtained by Fp2 and F4 electrodes for both arousal and valence dimensions. The F4 electrode is found to be sensitive for both arousal and valence dimension. The Fpl electrode yields maximum F-1 score for arousal while for valence it is shown by F4. The selected electrodes from the decision fusion based method yields better performance for classification of arousal and valence emotional states. It is observed that the frontal region electrodes (F3 and F4) are active in both arousal and valence dimensions.

\section{Conclusion}

In this study, EEG signals in the frontal region are analyzed using time-domain features. It is observed that the different electrodes have different responses to the same stimuli, and features are different for different arousal and valence class. Further, the information fusion using DS evidence-based theory is used for the electrode selection. Theoretically it was established that the frontal electrodes are useful for emotion classification. The results help to find out the best electrodes from the frontal electrodes for classification of arousal and valence emotional states. This method can further be used for selecting electrodes from all the channels and with multiple features to achieve further better performance.

\section{References}

[1] Reisenzein R. What is a definition of emotion? And are emotions mental-behavioral processes?. Social science information. 2007 Sep;46(3):424-8.

[2] Posner J, Russell JA, Peterson BS. The circumplex model of affect: An integrative approach to affective neuroscience, cognitive development, and psychopathology. Development and psychopathology. 2005;17(3):715.

[3] Teplan M. Fundamentals of EEG measurement. Measurement science review. 2002 Jan;2(2):1-1.

[4] Umeda S. Emotion, personality, and the frontal lobe. InEmotions of Animals and Humans 2012; Springer, Tokyo. p. 223-241.

[5] Jenke R, Peer A, Buss M. Feature extraction and selection for emotion recognition from EEG IEEE Trans Affective Comput. 2014 Jul 17;5(3):327-39.

[6] Leung Y, Ji NN, Ma JH. An integrated information fusion approach based on the theory of evidence and group decision-making. information fusion. 2013 Oct 1;14(4):410-22.

[7] Koelstra S, Muhl C, Soleymani M, Jong-Seok Lee, Yazdani A, Ebrahimi T, et al. DEAP: A Database for Emotion Analysis ;Using Physiological Signals. IEEE Trans Affective Comput. 2012 Jan;3(1):1831.

[8] Ahirwal MK, Kose MR. Audio-visual stimulation based emotion classification by correlated EEG channels. Health and Technology. 2020 Jan;10(1):7-23.

[9] Hjorth B. EEG analysis based on time domain properties. Electroencephalography and clinical neurophysiology. 1970 Sep 1;29(3):306-10.

[10] Accardo A, Cusenza M, Monti F. Linear and non-linear parameterization of EEG during monitoring of carotid endarterectomy. Computers in biology and medicine. 2009 Jun 1;39(6):512-8.

[11] Nawaz R, Cheah KH, Nisar H, Voon YV. Comparison of different feature extraction methods for EEGbased emotion recognition. Biocybernetics and Biomedical Engineering. 2020 May 11.

[12] Luo C, Wang Z, Wang S, Zhang J, Yu J. Locating facial landmarks using probabilistic random forest. IEEE Signal Processing Letters. 2015 Sep 22;22(12):2324-8.

[13] Pan Y, Zhang L, Wu X, Skibniewski MJ. Multi-classifier information fusion in risk analysis. Information Fusion. 2020 Feb 25. 